\title{
Application of Artificial Intelligence in Power System Fault Diagnosis
}

\author{
Jing Liu ${ }^{1, a}$ Wenbo Jiang*1, b ${ }^{{ }^{1,}}$ Li Miao ${ }^{1, \text { c }}$, Jian Zhang ${ }^{2, \text { d }}$ \\ ${ }^{1}$ Haikou College of Economics, Haikou, Hainan, 571127 \\ ${ }^{2}$ Hainan University, Haikou, Hainan, 570228 \\ ${ }^{a}$ email, ${ }^{b}$ email, ${ }^{c}$ email, ${ }^{d}$ email
}

Keywords: Artificial Intelligence, Power System, Fault Diagnosis

\begin{abstract}
In the modern society, the economic development of our country is moving rapidly and the material living standards of the people are constantly increasing. As a result, the power resources occupy an increasingly important position in people's daily life. However, because of this, people's daily life In the electricity safety issues are also more and more attention. The power companies in the power system fault diagnosis should also put more effort, the only way to ensure people's daily life of electricity is safe and reliable, based on this background, but also with the continuous progress of artificial intelligence in recent years, as well as in Power system fault diagnosis has a significant role. Therefore, this article analyzes the practical application of artificial intelligence technology in power system fault diagnosis, and explores in all aspects the great impact of artificial intelligence technology on power failure detection.
\end{abstract}

\section{Introduction}

The development of science and technology has driven the gradual popularization of smart life in all aspects of society. At the present stage of our country, we are vigorously promoting the implementation of smart grid, making the modern power system move forward towards informationization and intelligence. Under these preconditions, The role of power system construction can be very important. As a part of people's daily life, the power system is very important for the stability and safety. If the power system fails due to various reasons, it will directly affect people's normal production and survival. Similarly, if the stable operation of the power system can not be timely fault diagnosis, it will lead to failure problems can not be promptly confirmed and dealt with, resulting in unnecessary losses. In order to avoid this phenomenon, applying various practical methods of artificial intelligence to detect daily power system faults is the key to the detection and repair of the power system after the studio. Next we analyze the specific meaning of artificial intelligence technology and practical application. With the popularization of artificial intelligence technology, it plays a vital role in modern power system. In the various stages of daily operation of power system, expert system (ES), artificial neural network Artificial Intelligence (ANN), Fuzzy Theory (FT), Genetic Algorithmic (GA) and other artificial intelligence techniques have been extensively applied to power operations such as production control, supervision and management, and fault detection. Intelligent detection system in modern power system which mainly refers to, relying on artificial intelligence as the theoretical basis for professional knowledge and related technical means to power equipment in various intelligent fault, information supervision and management. Why Artificial Intelligence Systems are widely recognized nowadays mainly because of the variety of causes of faults that occur in normal power system fault diagnosis work, and safety testers are prone to errors in actual fault detection. Therefore, Artificial Intelligence Can help solve these problems, so as to ensure the rational use of resources and save manpower and resources. Here, the article focuses on the most comprehensive intelligent FD-PS in the international power failure diagnosis system market summarized and analyzed, mainly on the ES, ANN, FST, GA and Petri networks and other technologies in the FDPS in the practical application of finishing. ES-based power system fault diagnosis ES principle refers to the combination of relevant professional books on the power of theoretical knowledge, combined with the power supervision and management experts and staff of the actual operating 
experience to a variety of power failure problems of science Reasonable treatment. For those problems that simply can not be resolved by common analytical methods, ES is just an expression that can effectively reduce the scope of the cause of the failure problem and help to improve work efficiency. On the other hand, ES reasoning can also explain to a large extent Help power system fault detection and diagnosis of efficient and orderly operation. The main applications of ES in power system fault diagnosis are as follows: predicate logic representation, production rule representation, procedural knowledge representation, frame-based representation, Knowledge model representation is based on object-oriented representations. Among these representations are predicate-based logical notation, production-based rule notation, and procedural knowledge-based representations that are more common than conventional ones. Knowledge-based representations and object- There is also based on object-oriented notation, it is for the above method of extending and extending is based on the formation of the development of new forms of expression and new expression. Next, we systematically analyze the specific application of these expressions in store fault detection: Based on predicate logic representation can be said to be a relatively early expression of professional knowledge than other expressions to describe the way, the main The way it works is to build a proprietary power knowledge base using protection and circuit breaker information and then construct several knowledge bases for predicate logic using the Prolog language. The first is a description of the system that shows the specific structure of the power system, focusing on expertise in protection and circuit breaker actuation, and the correct description of the state of the circuit breaker. The second is mainly to emphasize how power systems Protection principle to carry out a comprehensive and correct description of the expression; the third is to present the actual troubleshooting work to describe the law of the location of the daily fault, as well as the use of reverse reasoning method combined with the above fault equipment and Protection, circuit breaker information to complete the diagnosis of the failure of the operation of the resistance factor processing. Although the method is simple and effective, the actual production process is too cumbersome and inefficient is the biggest limitation of predicate logic.

\section{Application Analysis of Artificial Intelligence in Power System Fault Diagnosis}

Fault diagnosis of information is indefinite, mainly due to protection devices, circuit breakers refuse to move or damaged during the transmission of information and other reasons. For this reason, researchers have published some uncertain information in the field of fault diagnosis. The introduction of the theory of uncertainty is based on the fact that the fault information is influenced by many factors, and usually unsafe colors. The basic artificial intelligence technology mainly includes fuzzy theory, probability theory, rough set and so on. The theory of fuzzy is about the expert, but he prolongs the time in the process of practical work, in the practical ability, usually combined with other methods, the fuzzy information and the expert's complete system diagnosis fault, calculates the uncertainty of the transmission using Petri net and After the termination of the network is completed, although there is a certain effect, but the understanding and control of the practical application and maintenance of learning and function of the hard work to determine the members of the change-related issues are still in the research stage and other factors, the application is still limited of. Probability theory is divided into theory of theoretical Bayesian reliability, probability of information representation as a preliminary or power system fault, and the actual implementation of belief propagation by generating rule-based information on the values of the belief that the method is based on a combination of probabilistic Petri nets. The effect is obvious, but due to its dependence on some informational experience, the formula used to solve the event theory autonomously decides the roughness of the energy system, as a class attribute defenses certain devices and switches in combination with a fault table may be impractical decision control. The original set of information to reduce the role of the principle algorithm, the original too, to minimize the minimization of rough set to minimize the decision-making. However, the real failure to make a diagnosis, its practical application at this stage, it is difficult to affect the formation of a major decision of the current table is a pleasing effect.

Based on computer technology and computer science, process optimization equates to the 
infinite optimization and global optimization algorithms that solve this problem, and the continuous development of artificial intelligence techniques for zero programming. For example, fault diagnosis diagnoses the system fault energy, and the relationship between fault diagnosis based on the use of switch protection genetic algorithm or mathematical theory can be seen as based on the problem of using integer programming that can be solved before and after changing the network topology. Problem Solving Network Annealing Algorithm for Effective Reduction Based on the number of media solutions that configure the vehicle and fault information, a diagnostic fault is established to simulate a model fault diagnosis. However, it is difficult to establish a reasonable model for fault diagnosis in practical applications, and there are many random factors in order to achieve accurate and rapid fault diagnosis of power system.

The combination of multiple approaches to artificial intelligence based on the fusion of multiple approaches is based on the idea that the failure of the power system is complex and less comprehensive if only a single diagnostic approach is taken. For example, in combination with neural network methods and fuzzy logic, overall performance practices significantly improve the ability of Petri nets to integrate self-taught information in order to improve the self-taught ability of neural networks and algorithms that can be combined with genetic algorithms and expert systems to Improve the speed of power failure diagnosis system. And his performance anti-interference ability, we can see that in a variety of power system fault diagnosis work, artificial intelligence technology integration method is one of the main directions of its future development.

\section{Application and Analysis of Artificial Intelligence Based on Optimization Technology in Power System Fault Diagnosis}

Based on the continuous development of computer technology and computational science, the artificial intelligence technology based on optimization technique is equivalent to the unconstrained 0 to 1 integer programming problem in power system fault diagnosis, Its mathematical theory as a support, so the theory and practicality are more ideal, for example, fault diagnosis in the power system to determine the components and protection of the relationship between the action of the protection switch, fault diagnosis can be considered as an integer programming based on the use of Genetic algorithm, or annealing algorithm. In the process of solving, the data model of the fault area can be established according to the change of the network topology before and after the system failure, so as to effectively reduce the scale of the solution. When multiple solutions occur, Combined with the characteristics of the secondary configuration and the fault diagnosis of a new diagnostic model simulation, but in practical applications by the establishment of a reasonable fault diagnosis model is more difficult, and there are many factors such as the impact of random factors, the current power system fault diagnosis and application Not extensive.

\section{Conclusion}

Through the above analysis, it can be found that the application of artificial intelligence technology to power system fault diagnosis is determined by its own characteristics and power system fault diagnosis, which is the inevitable choice for the development of power system in our country. However, The application of fault diagnosis has its own advantages and disadvantages, in the specific application process should be combined with the actual situation, to do specific analysis of specific problems in order to achieve accurate and rapid diagnosis of fault for the direct purpose.

\section{Acknowledgements}

Project Title (Level): Hainan Province, higher education and teaching reform research key project "Internet of Things construction and wisdom of Hainan research and practice"

Project Number: Hnjg2016ZD-22 


\section{References}

[1] Talent only bright. Application of artificial intelligence in power system fault diagnosis [J]. Guangdong Electric Power, 2011,9: 87-92.

[2] Wang Lei. Grid fault diagnosis method and its system architecture [D]. Shandong University, 2013.

[3] Wu Xin. Power system fault diagnosis based on improved Bayesian network method [D]. Zhejiang University, 2005.

[4] Zhu Lijuan, Fang Qian. Application of Intelligent Technology in Power System Fault Diagnosis [J]. Urban Geography, 2014,18: 198.

[5] Wang Lei. Grid fault diagnosis method and its system architecture [D]. Shandong University, 2013. 